\title{
Thermodynamic properties and phase diagrams of spin-1 quantum Ising systems with three-spin interactions
}

\author{
Hui Jiang and Xiang-Mu Kong** \\ College of Physics and Engineering, \\ Qufu Normal university, Qufu 273165, China
}

\begin{abstract}
The spin-1 quantum Ising systems with three-spin interactions on two-dimensional triangular lattices are studied by mean-field method. The thermal variations of order parameters and phase diagrams are investigated in detail. The stable, metastable and unstable branches of the order parameters are obtained. According to the stable conditions at critical point, we find that the systems exhibit tricritical points. With crystal field and biquadratic interactions, the system has rich phase diagrams with single reentrant or double reentrant phase transitions for appropriate ranges of the both parameters.
\end{abstract}

PACS numbers: $64.60 . \mathrm{Kw}, 64.60 . \mathrm{Cn}, 05.70 . \mathrm{Fh}, 75.10 . \mathrm{Jm}$

Keywords: Three-spin interactions; Crystal field; Phase diagram; Tricritical point; Reentrant

${ }^{*}$ Corresponding author; Electronic address: kongxm@mail.qfnu.edu.cn (X.-M. Kong) 


\section{INTRODUCTION}

The models with multispin interactions have attracted much attentions, such as the eight-

vertex model [1, 2] which involves both two- and four-spin interactions, the Ising model with four-spin interactions [3, 4] and the Baxter-Wu model [5] which is a non-trivial Ising model with three-spin interactions on a triangular lattice. The Hamiltonian of the Baxter-Wu model is

$$
H=-J \sum_{\langle i j k\rangle} \sigma_{i} \sigma_{j} \sigma_{k}
$$

where $\langle i j k\rangle$ denotes a triplet of nearest neighbor sites of a triangular lattice, $J$ is the coupling constant, and $\sigma_{i}= \pm 1$ is the Ising spin located at site $i$. Besides the two-dimensional Ising model [6], the Baxter-Wu model is another exactly solvable one which exhibits spontaneous symmetry breaking. The system has been studied by series-expansion analysis [7] 9], Monte Carlo [9-11], Monte Carlo renormalization group [12, 13] and conformal invariance approaches [14, 15], etc. It has the same critical temperature as the Ising model with three-spin interactions in one direction on a square lattice [16], and belongs to the same universality class as two-dimensional 4-state Potts system with the equal critical exponents [17, 18], namely, their specific heat critical exponent and the correlation length exponent are equal to $2 / 3(\alpha=\nu=2 / 3)$, and correlation function exponent is $\eta=1 / 4$. In addition, Costa et al. [19] have studied the spin-1 Baxter-Wu model with a crystal field and found that the phase diagram presents a tricritical point.

In 1990s, due to describing incommensurate phases of quantum one-dimensional magnetics, Tsvelik constructed a modified XXZ model [20] with Hamiltonian

$$
H=\sum_{n=1}^{N} \sigma_{n} \cdot \sigma_{n+1}+J_{2} \sum_{n=1}^{N} \sigma_{n-1} \cdot\left(\sigma_{n} \times \sigma_{n+1}\right),
$$

where $\sigma_{i}^{\alpha}(\alpha=x, y, z)$ are pauli matrices, $N$ is the total number of spins, $J_{2}$ is the threespin interactions strength. With competing of the two and three-spin interactions, the model exhibits several quantum phase [21, 22]. In this paper, imitating Eq. (2) we define a quantum Ising model with three-spin interactions on a triangular lattice.

It is well-known that the Blume-Capel (BC) model [23-25] and the Blume-Emery-Griffiths (BEG) model [26] present much interesting critical phenomenon: tricritical point, multicritical phase diagrams [27-31], the reentrant phenomenon [31-33], metastable and unstable 
states [34, 35]. Similar to the BC model and BEG model, we study the spin-1 quantum Ising systems with a crystal field and biquadratic interactions by mean-field method. Our emphases are the thermodynamic properties and phase diagrams of the systems. We expect our system can exhibit that interesting proprieties.

The outline of this paper is as follows. In section III using the mean-field method, we study the spin-1 quantum Ising system with a crystal field. In Sec. III, the spin-1 quantum Ising system with biquadratic interactions is studied in detail, and Sec. IV is a brief summary.

\section{QUANTUM ISING SYSTEM WITH A CRYSTAL FIELD}

The spin-1 quantum Ising model with three-spin interactions in presence of a crystal field on a triangular lattice is defined by the Hamiltonian

$$
H=-J \sum_{\langle i j k\rangle} S_{i}^{z} \cdot\left(\vec{S}_{j} \times \vec{S}_{k}\right)^{z}+D \sum_{i}\left(S_{i}^{z}\right)^{2},
$$

where $\vec{S}_{i}=S_{i}^{x} \vec{i}+S_{i}^{y} \vec{j}+S_{i}^{z} \vec{k}, S_{i}^{\alpha}(\alpha=x, y, z)$ are components of the spin-1 operator at site $i$, and $D$ plays the role of a crystal field. $S^{\alpha}(\alpha=x, y, z)$ are

$$
S^{x}=\frac{\sqrt{2}}{2}\left(\begin{array}{lll}
0 & 1 & 0 \\
1 & 0 & 1 \\
0 & 1 & 0
\end{array}\right), S^{y}=\frac{\sqrt{2}}{2}\left(\begin{array}{lll}
0 & -i & 0 \\
i & 0 & -i \\
0 & i & 0
\end{array}\right), S^{z}=\left(\begin{array}{lll}
1 & 0 & 0 \\
0 & 0 & 0 \\
0 & 0 & -1
\end{array}\right)
$$

In the following discussion, the coupling constant $J>0$, that is, a ferromagnetic case is considered. Based on the mean-field approximation [36, 37], the three-spin cluster Hamiltonian $H_{123}$ can be written as

$$
H_{123}=-J S_{1}^{z} \cdot\left(\vec{S}_{2} \times \vec{S}_{3}\right)^{z}+D\left(\left(S_{1}^{z}\right)^{2}+\left(S_{2}^{z}\right)^{2}+\left(S_{3}^{z}\right)^{2}\right)-4 J m\left(S_{1}^{z}+S_{2}^{z}+S_{3}^{z}\right)
$$

where $m$ is the average magnetization of the cluster, (i.e., $\left.m=\left\langle\left(S_{1}^{z}+S_{2}^{z}+S_{3}^{z}\right) / 3\right\rangle\right)$. In the representation of the direct product of $S_{1}^{z}, S_{2}^{z}$ and $S_{3}^{z}, H_{123}$ can be written as a form of $27 \times 27$ matrix. Thus the partition function $Z=\operatorname{Tr}\left[\exp \left(-\beta H_{123}\right)\right]\left(\beta=\frac{1}{k_{B} T}\right.$, where $k_{B}$ is the Boltzmann constant, $T$ denotes the temperature of the system) can be obtained as

$$
Z=1+4 a_{1} \cosh (4 K m)^{2}+4 a_{2} \cosh (4 K m)+2 e^{-3 K D_{0}} \cosh (12 K m)
$$

in which

$$
a_{1}=e^{-2 K D_{0}}(1+2 \cosh K)
$$




$$
a_{2}=e^{-2 K D_{0}}\left[2 \cosh \left(K D_{0}\right)+\cosh \left(K \sqrt{2+D_{0}^{2}}\right)\right],
$$

where $K=J / k_{B} T$ is the reciprocal of the reduced temperature, and $D_{0}=D / J$ is the reduced crystal-field parameter. The reduced free energy $f$ of the three-spin cluster in the system as a function of $K$ and $m$ is further given by

$$
f(m, K)=6 m^{2}-K^{-1} \ln [Z(m, K)] .
$$

According to the equilibrium condition of the system $(\partial f / \partial m=0)$, we can obtain the self-consistent equation of the magnetization

$$
m=\frac{2 a}{3 Z}
$$

where

$$
a=2 a_{1} \sinh (8 K m)+2 a_{2} \sinh (4 K m)+3 e^{-3 K D_{0}} \sinh (12 K m) .
$$

By solving the self-consistent Eq. (9), numerically, we obtain the thermal variations of the magnetization for several values of $D_{0}$ (Fig. 1). We obtain the stable, metastable and unstable branches, which are represented by the solid, dot and dash-dotted lines, respectively. It can be seen that for $D_{0}=1,1.6$ and 1.9 the stable magnetization are continuous, indicating the second-order phase transitions. For $D_{0}=1.6$ and 1.9, we also find unstable solutions. On the other hand, the stable magnetization drops discontinuously from a finite value $m_{\mathrm{F}}^{*}\left(D_{0}\right)$ to zero at a temperature $T_{\mathrm{F}}\left(D_{0}\right)$, which characterizes a first-order phase transition. The first-order phase transition temperature $T_{\mathrm{F}}\left(D_{0}\right)$ can be obtained by the free energy, when its local minimum at $m \neq 0$ is equal to the local minimum at $m=0$ [36, 37]. When the reduced crystal-field parameter is slightly larger than the tricritical point $D_{0}^{\operatorname{tr}}(1.95138)$, the system can undergo the first-order phase transition, such as, for $D_{0}=1.98$ the first-order phase transition temperature is $k_{B} T_{\mathrm{F}} / J \approx 0.84112$.

Figure 2 shows the phase diagram of the spin-1 quantum Ising system in the presence of a crystal field. The critical line separates the ferromagnetic phase from the paramagnetic phase. We can see that the second-order phase transition (solid line) occurs for finite values of the crystal field. The second-order phase transition temperature falls smoothly with the reduced crystal-field parameter $D_{0}$ increasing and reaches the tricritical point. The value of the tricritical point is $D_{0}^{\mathrm{tr}}=1.95138$ and $k_{B} T_{\mathrm{tr}} / J=1.1898$, which is different from that in ref [13] $D_{0}^{\mathrm{tr}}=1.3089$ and $k_{B} T_{\mathrm{tr}} / J=1.2225$. Then, the first-order phase transition line falls 
smoothly from the tricritical point to zero. This phenomenon is similar to the spin-1 BaxterWu model [19]. It need to clear that the last point of the curve in Fig. 2 is $D_{0}=2.00524999$ and $k_{B} T_{F} / J=0.4635712$.

\section{QUANTUM ISING SYSTEM WITH BIQUADRATIC INTERACTIONS}

\section{A. Model and formulation}

The Hamiltonian of the spin-1 quantum Ising system with biquadratic interactions on a triangular lattice is defined as

$$
H=-J \sum_{\langle i j k\rangle} S_{i}^{z} \cdot\left(\vec{S}_{j} \times \vec{S}_{k}\right)^{z}-G \sum_{\langle i j\rangle}\left(S_{i}^{z}\right)^{2}\left(S_{j}^{z}\right)^{2}+D \sum_{i}\left(S_{i}^{z}\right)^{2}
$$

where $\langle i j\rangle$ indicates all nearest-neighbor pairs of sites of a triangular lattice and $G$ denotes the biquadratic interactions parameter. Different from that in Sec. III, for solving this model, we need two order parameters: the magnetization $m$ and quadrupolar moment $q$. Using the similar method as in Sec. II the three-spin cluster Hamiltonian $H_{123}$ has the following expression:

$$
\begin{aligned}
H_{123}= & -J S_{1}^{z} \cdot\left(\vec{S}_{2} \times \vec{S}_{3}\right)^{z}-G\left(\left(S_{1}^{z}\right)^{2}\left(S_{2}^{z}\right)^{2}+\left(S_{2}^{z}\right)^{2}\left(S_{3}^{z}\right)^{2}+\left(S_{3}^{z}\right)^{2}\left(S_{1}^{z}\right)^{2}\right) \\
& +(D-4 G q)\left(\left(S_{1}^{z}\right)^{2}+\left(S_{2}^{z}\right)^{2}+\left(S_{3}^{z}\right)^{2}\right)-4 J m\left(S_{1}^{z}+S_{2}^{z}+S_{3}^{z}\right)
\end{aligned}
$$

where $m$ has been defined in Sec. II, $q$ is the average squared magnetization of the threespin cluster, $q=\left\langle\left(\left(S_{1}^{z}\right)^{2}+\left(S_{2}^{z}\right)^{2}+\left(S_{3}^{z}\right)^{2}\right) / 3\right\rangle$. According to the definition of the partition function, it is obtained as

$$
Z=1+2 b_{1} \cosh (12 K m)+4 b_{2} \cosh (4 K m)^{2}+4 b_{4} \cosh (4 K m)
$$

in which

$$
\begin{gathered}
b_{1}=e^{3 K\left(-D_{0}+G_{0}+4 q G_{0}\right)}, \\
b_{2}=e^{K\left(-2 D_{0}+G_{0}+8 q G_{0}\right)}(1+2 \cosh K), \\
b_{3}=\sqrt{8+\left(-2 D_{0}+(3+8 q) G_{0}\right)^{2}}, \\
b_{4}=b_{1}+e^{-K\left(D_{0}-4 q G_{0}\right)}+e^{K\left(-2 D_{0}+3 G_{0} / 2+8 q G_{0}\right)} \cosh \left(K b_{3} / 2\right),
\end{gathered}
$$


where $G_{0}=G / J$ is the reduced biquadratic parameter. The reduced free energy of the three-spin cluster can be written as

$$
f\left(K, G_{0}, D_{0}, m, q\right)=6 m^{2}+6 G_{0} q^{2}-K^{-1} \ln Z\left(K, G_{0}, D_{0}, m, q\right) .
$$

With the equilibrium conditions $(\partial f / \partial m=0$ and $\partial f / \partial q=0)$ of the system, the selfconsistent equations of the two order parameters are

$$
m=\frac{2 g}{3 Z}
$$

and

$$
q=\frac{2 h}{3 Z}
$$

in which

$$
\begin{gathered}
g=3 b_{1} \sinh (12 K m)+2 b_{2} \sinh (8 K m)+2 b_{4} \sinh (4 K m), \\
h=3 b_{1} \cosh (12 K m)+4 b_{2} \cosh (4 K m)^{2}+2\left(2 b_{1}+b_{4}+b_{5}\right) \cosh (4 K m),
\end{gathered}
$$

where

$$
b_{5}=e^{K\left(-2 D_{0}+3 G_{0} / 2+8 q G_{0}\right)}\left[\cosh \left(K b_{3} / 2\right)+\sinh \left(K b_{3} / 2\right)\left((3+8 q) G_{0}-2 D_{0}\right) / b_{3}\right] .
$$

\section{B. Results and Discussions}

By numerically calculating Eqs. (19) and (20), the behavior of the magnetization $m$ and quadrupolar moment $q$ as functions of temperature are obtained (see Figs. 3 and 4). We obtain the stable (M1 and Q1), unstable (M2 and Q2) and metastable (M3 and Q3) branches. As in Sec. II, the stable branches of the order parameters undergo the first-order or secondorder phase transition. What is interesting is that in Figs. 3(d) and 3(e), the stable branches of the order parameters undergo one first-order phase transition and one second-order phasetransition successively. Fig. 4(b) shows that the stable branches of the order parameters undergo two second-order phase transitions and one first-order phase transition. Otherwise, what should be mentioned is that the stable branch of the quadrupole order parameter does not undergo any phase transition when the stable branch of the magnetization is equal to zero [35] (see Fig. 3(f)). Seen from Fig. 3, at $T=0$, the system can be in the ground state with $m=q=1, m=q=2 / 3$ or $m=q=0$, which is similar to the ground state of 
the $S=1$ Ising model on triangular lattice [38]. The actual ground state depends on the competition between the reduced biquadratic interaction and crystal field.

Figure 5 shows three phase diagrams for different biquadratic parameter $G_{0}$. It can be seen that the first-order phase transition can occur either in the range of $D_{0}<D_{0}^{\operatorname{tr}}$ (Fig. 5(a)) or $D_{0}>D_{0}^{\text {tr }}$ (Fig. 5(c)). At fixed reduced biquadratic and crystal-field parameter in Fig. 5(a), when the temperature decrease the transition from paramagnetic phase $(\mathrm{P})$ to ferromagnetic phase $(\mathrm{F})$ is encountered, and if the temperature is lowered further, the reentrant phase transition from ferromagnetic phase to paramagnetic phase can take place. Fig. 5(c) shows a double reentrant phenomena, that is, at fixed reduced biquadratic and crystal field parameter, as the temperature decrease, the $\mathrm{P} \rightarrow \mathrm{F} \rightarrow \mathrm{P} \rightarrow \mathrm{F}$ sequence of phase is encountered. It may be determined by the competition among quantum effect, crystal-field and the biquadratic interaction.

\section{SUMMARY}

In this paper, we have investigated the spin-1 quantum Ising systems with three-spin interactions on two-dimensional triangular lattices by mean-field approximation. The thermal variations of the magnetization $m$ and quadrupolar moment $q$ are obtained. By comparing the free energy of these solutions, we obtain the stable, unstable and metastable branches of the order parameters. In addition, we find that the quantum Ising systems present tricritical points for finite values of the crystal field, and the system with biquadratic interactions exhibits single reentrant and double reentrant phase transitions.

\section{Acknowledgments}

This work was supported by the National Natural Science Foundation of China under Grant No. 10775088, the Shandong Natural Science Foundation (Grant No. Y2006A05), and the Science Foundation of Qufu Normal University.

[1] R. J. Baxter, Phys. Rev. Lett. 26 (1971) 832.

[2] L. P. Kadanoff and F. J. Wegner, Phys. Rev. B 4 (1971) 3989. 
[3] J. Oitmaa and R. W. Gibberd, J. Phys. C 6 (1973) 2077.

[4] J. Oitmaa, J. Phys. C 7 (1974) 389.

[5] R. J. Baxter and F. Y. Wu, Phys. Rev. Lett. 31 (1973) 1294.

[6] L. Onsager, Phys. Rev. 65 (1944) 117.

[7] H. P. Griffiths and D. W. Wood, J. Phys. C 6 (1973) 2533.

[8] M. G. Watts, J. Phys. A 7 (1974) L85.

[9] J. Adler and D. Stauffer, Physica A 181 (1992) 396.

[10] M. A. Novotny and D. P. Landau, Phys. Rev. B 32 (1985) 5874.

[11] N. Schreiber and J. Adler, J. Phys. A 38 (2005) 7253.

[12] M. A. Novotny, D. P. Landau, and R. H. Swendsen, Phys. Rev. B 26 (1982) 330.

[13] M. A. Novotny and D. P. Landau, Phys. Rev. B 32 (1985) 3112.

[14] F. C. Alcaraz and J. C. Xavier, J. Phys. A 30 (1997) L203.

[15] F. C. Alcaraz and J. C. Xavier, J. Phys. A 32 (1999) 2041.

[16] J. M. Debierre and L. Turban, J. Phys. A 16 (1983) 3571.

[17] F. Y. Wu, Rev. Mod. Phys. 54 (1982) 235.

[18] R. J. Baxter, Exactly Solved Models in Statistical Mechanics, Academic, New York, 1982.

[19] M. L. M. Costa, J. C. Xavier, and J. A. Plascak, Phys. Rev. B 69 (2004) 104103.

[20] A. M. Tsvelik, Phys. Rev. B 42 (1990) 779.

[21] H. Frahm, J. Phys. A 25 (1992) 1417.

[22] P. Lou, W.-C. Wu, and M.-C. Chang, Phys. Rev. B 70 (2004) 064405.

[23] M. Blume, Phys. Rev. 141 (1966) 517.

[24] H. W. Capel, Physica 32 (1966) 966; 33 (1967) 295; 37 (1967) 423.

[25] D. M. Saul, M. Wortis, and D. Stauffer, Phys. Rev. B 9 (1974) 4964.

[26] M. Blume, V. J. Emery, and R. B. Griffiths, Phys. Rev. A 4 (1971) 1071.

[27] A. N. Berker and M. Wortis, Phys. Rev. B 14 (1976) 4946.

[28] R. R. Netz and A. N. Berker, Phys. Rev. B 47 (1993) 15019.

[29] A. Falicov and A. N. Berker, Phys. Rev. Lett. 76 (1996) 4380.

[30] A. Maritan, M. Cieplak, M. R. Swift, F. Toigo, and J. R. Banavar, Phys. Rev. Lett. 69 (1992) 221.

[31] W. Hoston and A. N. Berker, Phys. Rev. Lett. 67 (1991) 1027.

[32] O. R. Baran and R. R. Levitskii, Phys. Rev. B 65 (2002) 172407. 
[33] N. Seferoğlu and B. Kutlu, Physica A 374 (2007) 165.

[34] S. Lapinskas and A. Rosengren, Phys. Rev. B 49 (1994) 15190.

[35] C. Ekiz and M. Keskin, Phys. Rev. B 66 (2002) 054105.

[36] J. R. de Sousa, D. F. de Albuquerque, and I. P. Fittipaldi, Phys. Lett. A 191 (1994) 275.

[37] G.-H. Sun, and X.-M. Kong, Physica A 370 (2006) 585.

[38] J. B. Collins, P. A. Rikvold, and E. T. Gawlinski, Phys. Rev. B 38 (1988) 6741. 
Fig. 1 The magnetization $m$ versus temperature $k_{B} T / J$ for the spin-1 quantum Ising system with a crystal field on a triangular lattice. Curves a, b, c, d represent the stable branches for $D_{0}=1,1.6,1.9,1.98$, respectively. Curves b', c', d' represent the unstable branches and curve $\mathrm{d}^{\prime \prime}$ is the metastable branch.

Fig. 2 The phase diagram of spin-1 quantum Ising system with a crystal field on a triangular lattice. The solid line represents the second-order phase transition and dot line represents the first-order phase transition. The big real point (solid dot) is the tricritical point. $\mathrm{P}$ and $\mathrm{F}$ represent the paramagnetic and ferromagnetic phases, respectively.

Fig. 3 The magnetization $m$ and quadrupolar moment $q$ as functions of temperature $k_{B} T / J$ and crystal-field parameter $D_{0}$ with $G_{0}=-0.5$ for the spin-1 quantum Ising model with biquadratic interactions on a triangular lattice. M1, Q1 (solid line) are the stable branches of the magnetization $m$ and quadrupolar moment $q$, respectively; M2, Q2 (dot line) represent the unstable branches; M3, Q3 (dash-dot line) represent the metastable branches. $T_{\mathrm{F}}$ and $T_{\mathrm{C}}$ indicate the first-order and second-order phase transition temperature, respectively.

Fig. 4 The nonzero order parameters $m$ and $q$ as functions of temperature $k_{B} T / J$ and crystal-field parameter $D_{0}$ with $G_{0}=3$ for the spin-1 quantum Ising model with biquadratic interactions on a triangular lattice. M1, Q1 (solid line) represent the stable branches; M2, Q2 (dot line) represent the unstable branches. $T_{\mathrm{F}}$ and $T_{\mathrm{C}}$ indicate the first-order and second-order phase transition temperatures, respectively. $T_{\mathrm{C}}^{\prime}$ is the unstable critical temperature.

Fig. 5 Phase diagrams of the spin-1 quantum Ising model with biquadratic interactions on a triangular lattice for different $G_{0}$. The solid and dot lines represent the second-order and first-order phase transition lines and the solid dot represents the tricritical point. P and $\mathrm{F}$ denote the paramagnetic and ferromagnetic phase, respectively. (a) shows a $\mathrm{P} \rightarrow \mathrm{F} \rightarrow \mathrm{P}$ reentrant phase transitions. (c) shows a $\mathrm{P} \rightarrow \mathrm{F} \rightarrow \mathrm{P} \rightarrow \mathrm{F}$ double reentrant phase transitions. 


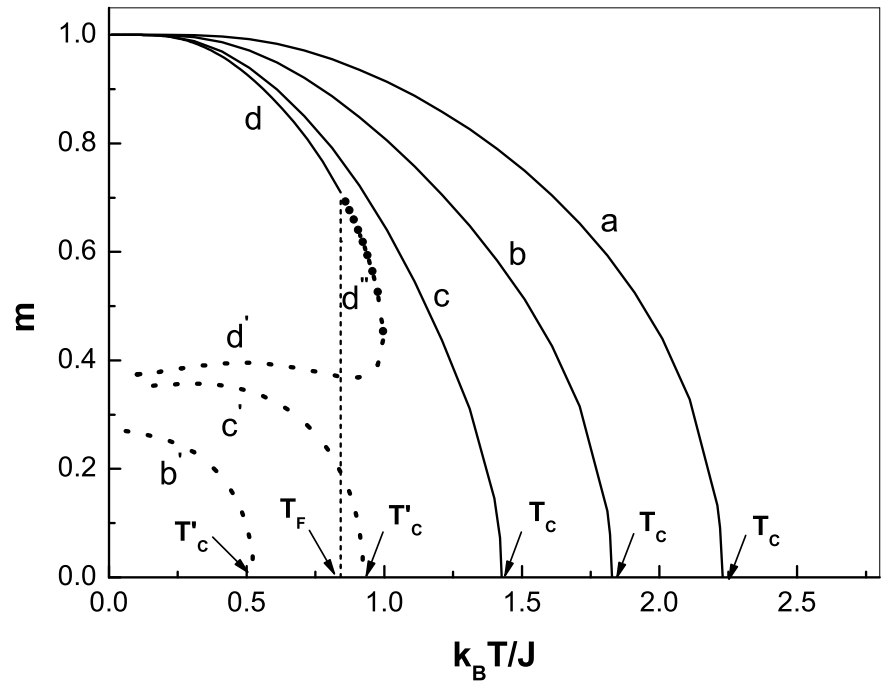




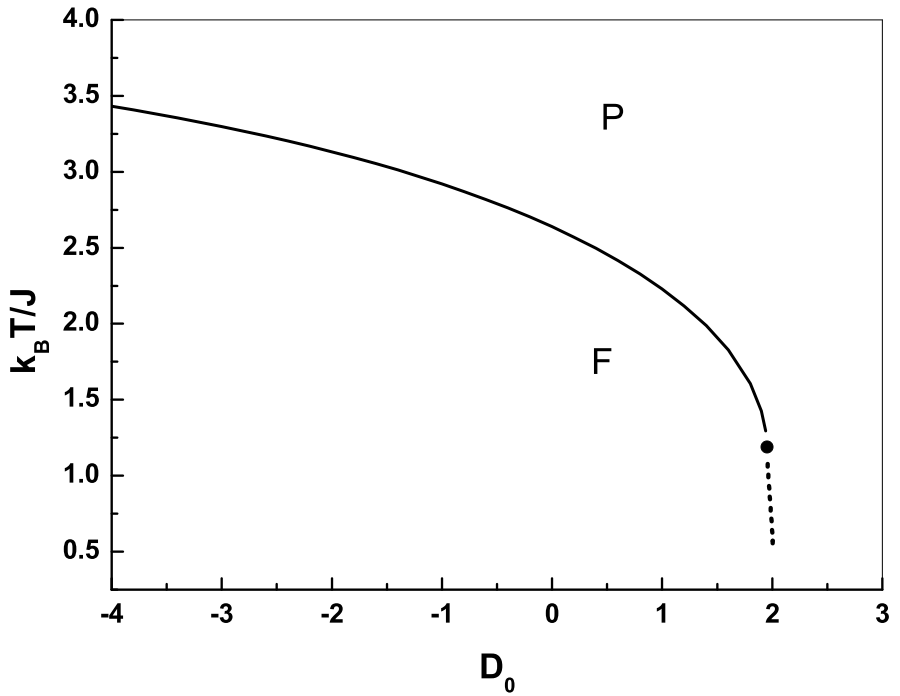



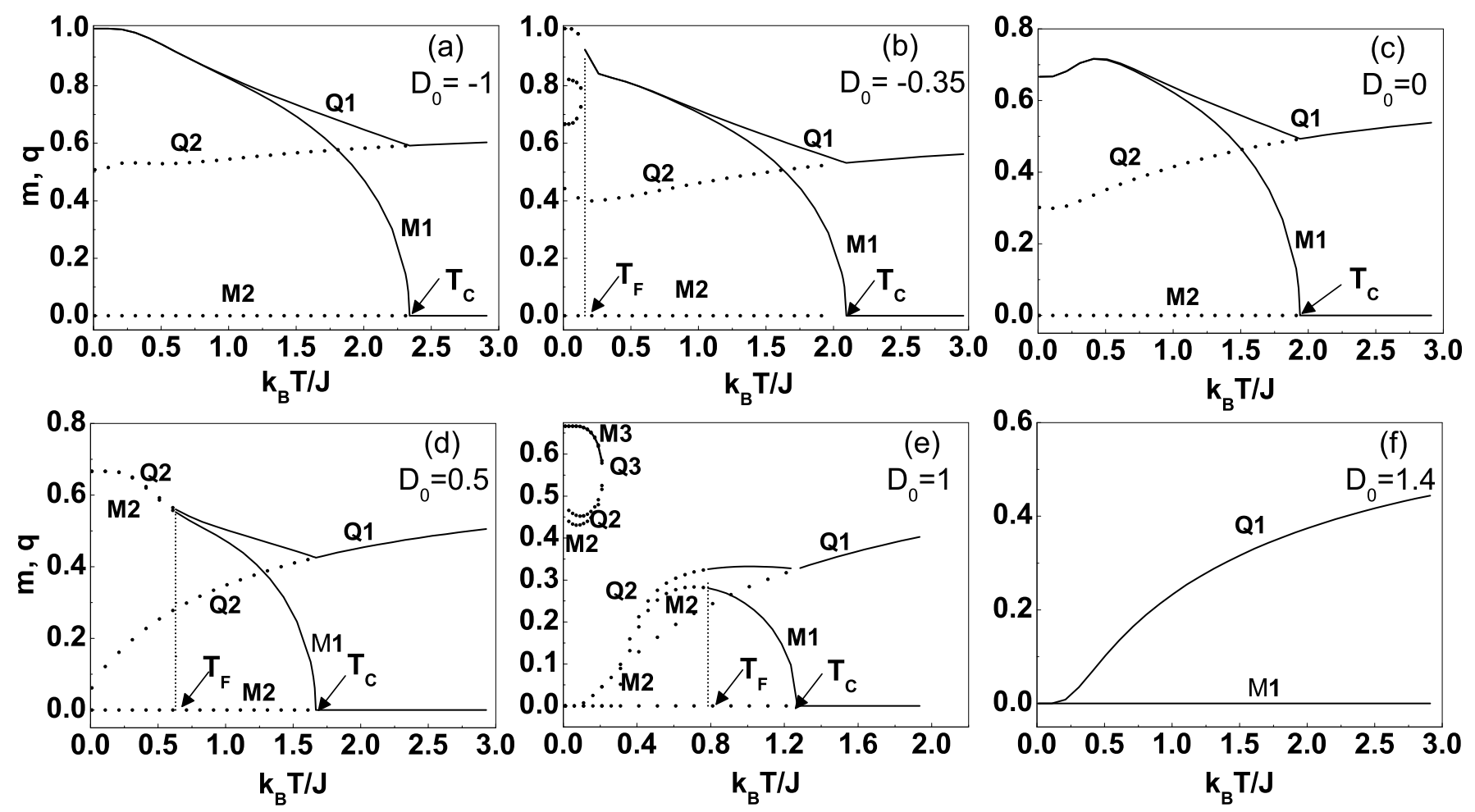

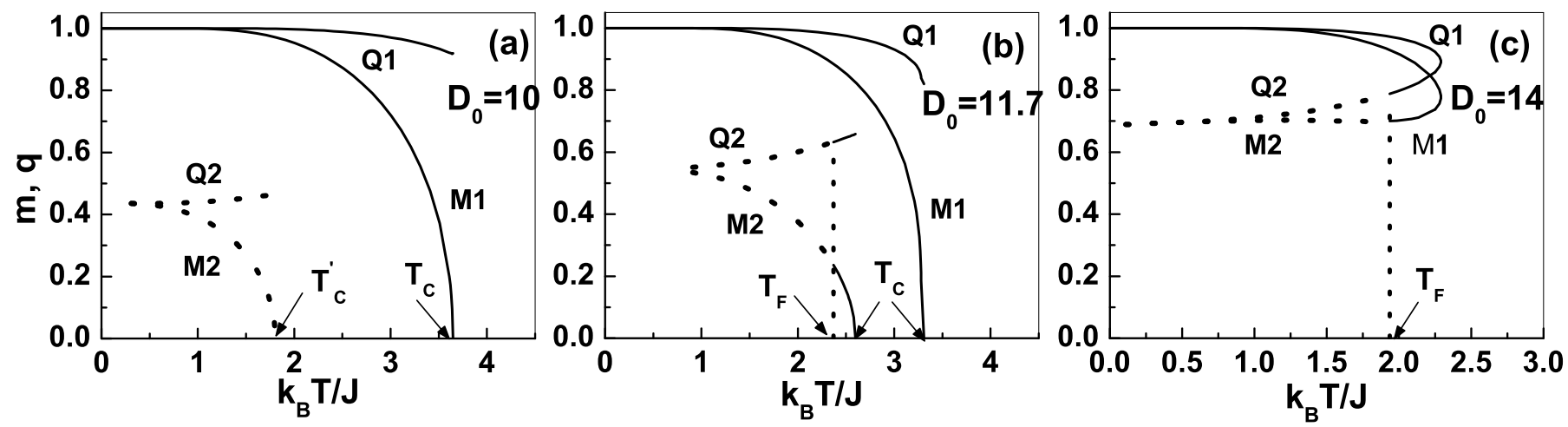

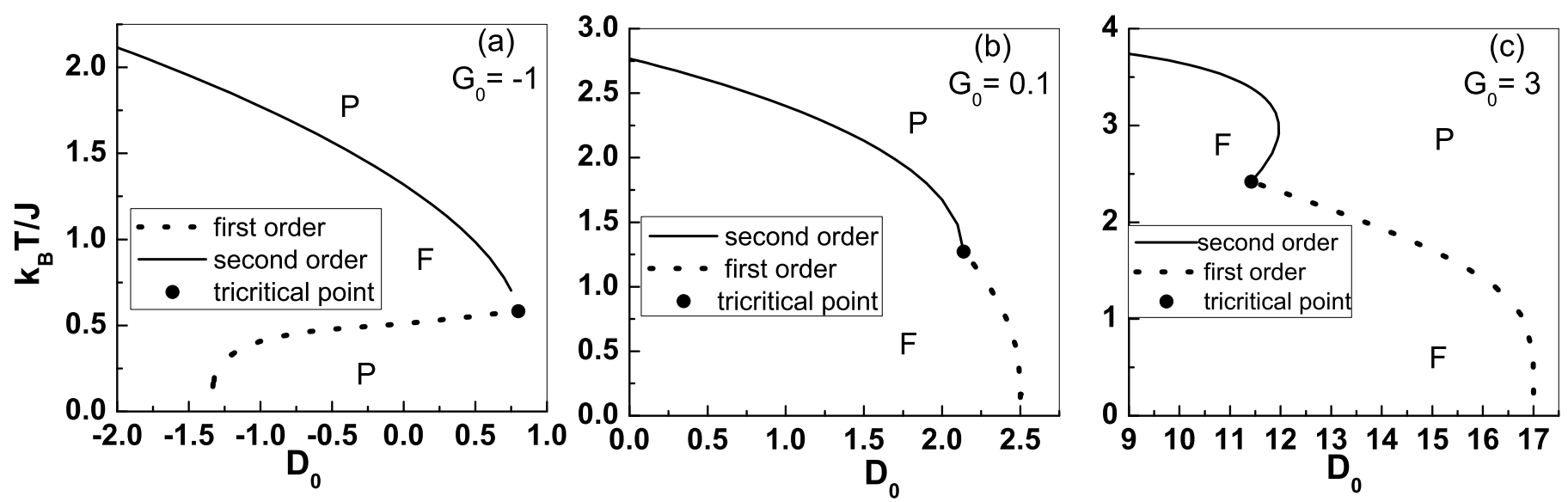